\title{
Susceptibilidad genética y enfermedad autoinmune reumática: Revisión narrativa
}

\author{
Genetic susceptibility and autoimmune rheumatic disease: Narrative review \\ Maynor Herrera-Méndez*1,2 \\ ${ }^{1}$ Unidad de Reumatología, Departamento de Medicina Interna, Hospital Roosevelt, \\ ${ }^{2}$ Escuela de Estudios de Postgrado, Facultad de Ciencias Médicas, Universidad de San Carlos \\ de Guatemala \\ *Autor al que se dirige la correspondencia: drherreram@gmail.com
}

Recibido: 04 de mayo 2020 / Revisión: 10 de junio 2020 / Aceptado: 30 de octubre 2020

\section{Resumen}

T a autoinmunidad es la consecuencia de la pérdida de control y regulación de la respuesta inmune. Se re-

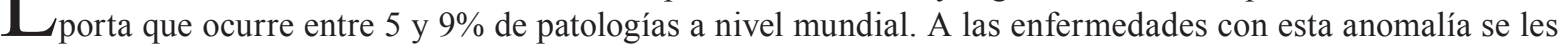
denomina autoinmunes y se clasifican de acuerdo con el órgano o sistema afectado. Las reumáticas involucran al tejido conectivo y las articulaciones. Los factores asociados a su aparición incluyen: edad, género, medioambiente y genéticos. La susceptibilidad genética indica la presencia de uno o varios genes asociados al desarrollo de determinada enfermedad, cuya expresión podría ser el producto de la migración, selección, recombinación y adaptación de genes entre las poblaciones, lo que explica la variación fenotípica y la expresión clínica resultante. Los estudios de asociación del genoma completo (GWAS por sus siglas en inglés) han permitido identificar múltiples genes involucrados con enfermedades reumáticas, destacan el lupus eritematoso sistémico y artritis reumatoide, asociadas con más de 60 alelos, y otras como la espondilitis anquilosante, en donde la asociación ha sido primordialmente con un gen y sus polimorfismos. Esta revisión tiene como objetivo informar el estado de la susceptibilidad determinada genéticamente para estas enfermedades y el impacto que tiene sobre la expresión clínica. Se realizó una búsqueda en PubMed y la base de datos de la biblioteca Cochrane, se incluyeron artículos relacionados con las palabras clave propuestas desde el 2000. La revisión identifica genes y la asociación con estas enfermedades, expone la diversidad existente y justifica continuar la búsqueda de genes en todas las poblaciones.

Keywords: Enfermedad autoinmune reumática, lupus eritematoso sistémico, artritis reumatoide, susceptibilidad genética, GWAS

\begin{abstract}
$\mathrm{A}$ utoimmunity is the consequence of the loss of control and regulation of the immune response. It is reported that between 5 and $9 \%$ of pathologies occur worldwide. Diseases with this abnormality are called autoimmune and are classified according to the organ or system affected. Rheumatic diseases involve connective tissue and joints. Factors associated with its appearance include age, gender, environment, and genetics. Genetic susceptibility indicates the presence of one or more genes associated with the development of a certain disease, whose expression could be the product of migration, selection, recombination and adaptation of genes between populations, which explains the phenotypic variation and the resulting clinical expression. Genome wide association studies (GWAS) have allowed the identification of multiple genes involved with rheumatic diseases, including systemic lupus erythematosus and rheumatoid arthritis, associated with more than 60 alleles, and others such as ankylosing spondylitis, where the association has been primarily with a gene and its polymorphisms. This review aims to report the status of genetically determined susceptibility to these diseases and the impact it has on clinical expression. A search was carried out in PubMed and the Cochrane library database, articles related to the proposed keywords from the year 2000 were included. The review identifies genes and the association with these diseases, exposes the existing diversity and justifies continuing the search for genes in all populations.
\end{abstract}

Palabras claves: Rheumatic autoimmune disease, systemic lupus erythematosus, rheumatoid arthritis, genetic susceptibility, GWAS 


\section{Introducción}

En la patogenia de las enfermedades reumáticas autoinmunes (ERA), el principal mecanismo patogénico es la pérdida de control sobre la respuesta inmune, lo que provoca el reconocer a antígenos propios como extraños, lo que induce la activación de la respuesta humoral y celular, la acumulación de linfocitos auto reactivos y la producción de auto anticuerpos, que desencadena una respuesta inflamatoria generalizada (Javinani et al., 2019).

Se desconoce cómo se inicia el desequilibrio de la respuesta inmune, se ha propuesto que pudieran estar involucrados genes, hormonas y principalmente factores del medio ambiente, el impacto de estos últimos induciría cambios en la expresión genética (Selmi Leung et al., 2012).

Inicialmente se pensaba que el fenómeno de autoinmunidad era provocado por la presencia de genes específicos, pero, con el advenimiento de técnicas genómicas y el desarrollo de la técnica de asociación del genoma completo (GWAS por sus siglas en inglés) se han encontrado múltiples genes relacionados (Deane \& Carlson, 2012).

La región del genoma que consistentemente está asociada y contribuye con el $30 \%$ de la susceptibilidad genética en todas las enfermedades autoinmunes, es el Complejo Mayor de Histocompatibilidad (CMH) ubicado en el cromosoma 6. Al conjunto de genes situados en esta área se les denomina genes del Antígeno del Leucocito Humano (HLA) por sus siglas en inglés; (Fernando et al., 2008).

La heterogeneidad étnica y genética contribuye a la complejidad de estas enfermedades, por lo que la búsqueda de genes implicados en la expresión de las ERA se ha extendido en las últimas décadas, y también se han encontrado genes en áreas fuera del $\mathrm{CMH}$ (especialmente en los cromosomas 1 y 10 y en otros con menor significancia), a los que se les ha llamado genes no HLA (Godkhe-Puranik \& Niewold, 2015).

La heredabilidad podría condicionar la aparición de enfermedades autoinmunes, en lupus eritematoso sistémico (LES) se ha reportado un índice de agregación $\lambda \mathrm{s}$ de $20 \%$ para hermanos, y $\lambda \mathrm{mz}$ de $60 \%$ para gemelos (Moser et al., 2009; Selmi, Lu et al., 2012) van Drongelen \& Holoshitz, 2017). Kuo y colaboradores (2017) han reportado un índice de heredabilidad para artritis reumatoide (AR) y otras enfermedades autoinmunes de $43.5 \%$ y un índice de transmisibilidad entre familiares de $59.4 \%$.
En familiares de primer grado el $8 \%$ de las mujeres tiene un familiar afectado por estas enfermedades, y hay asociación entre miembros de una familia entre 5-12\% (Alarcón-Segovia et al., 2005). Kuo y colaboradores (2017) reportan un riesgo relativo (RR) para el desarrollo de AR entre familiares de primer grado de 2.91 y para desarrollo de LES de 2.92.

Los factores medioambientales han sido relacionados con la pérdida de la auto tolerancia en sujetos que tienen predisposición genética, en las ERA la epigenética define los mecanismos implicados, siendo el más importante la hipometilación del ácido desoxirribonucleico (ADN) (Selmi, Lu et al., 2012).

Esta revisión tiene como objetivo informar $y$ delimitar, en la diversidad de genes, cuáles pueden ser de utilidad, no solo para el diagnóstico de estas enfermedades, si no en el futuro, para el tratamiento y pronóstico. Los criterios de búsqueda se centraron en las palabras susceptibilidad genética, enfermedad reumática autoinmune, patogénesis de la autoinmunidad, con filtros para estudios originales y revisiones sistemáticas (narrativas y metaanálisis), publicados a partir de 2000 y hasta la actualidad. Posteriormente se aplicó un segundo filtro para las palabras "lupus eritematoso sistémico" y "artritis reumatoide" que son las principales enfermedades reumáticas autoinmunes.

\section{Contenido}

\section{Mecanismos patogénicos de la autoinmunidad}

La primera descripción de autoinmunidad la realizó Paul Ehrlich en 1901: el horror autotoxicus, que debe entenderse como la pérdida de la regulación del sistema inmune, que dirige la respuesta inmunitaria hacia tejidos y órganos del propio organismo, lo que desencadena una enfermedad autoinmune; en la actualidad se conocen más de 78 de ellas, siendo las más importantes las que afectan el tejido conectivo y las articulaciones que se conocen como enfermedades reumáticas, las principales son: LES, AR, espondilitis anquilosante (EA), síndrome de Sjögren (SS) y las Miopatías inflamatorias (MI) entre otras. Las nuevas modalidades de recolección de información como es el caso del big data, en el futuro podrían aportar nueva información en relación con la frecuencia de estas enfermedades (Sisó-Almirall et al., 2020).

La pérdida de la auto tolerancia es el indicador principal de la desregulación de la respuesta inmune y 
puede ocurrir como consecuencia de factores reconocidos. De acuerdo con Javinani y colaboradores (Javinani et al., 2019), los principales factores serían genéticos y el medio ambiente, que inducirían la participación de células y moléculas del sistema inmune:

Genética. La susceptibilidad genética es la noción más aceptada, ya que la expresión de polimorfismos de algunos genes, codificarían proteínas que influirían negativamente sobre el control de la auto tolerancia y la metilación de ADN y la modificación de las histonas (principal aporte de la epigenética), especialmente los genes de IFN (interferón) relacionados estrechamente con los cambios en la metilación y la producción de auto anticuerpos.

Factores del medio ambiente. Entre los que sobresalen los agentes vivos (virus, bacterias, etc.) por los mecanismos de: mimetismo molecular, espectador inocente, infección persistente y la presencia de super antígenos; y los agentes inorgánicos como la radiación ultravioleta, hidrocarburos, pesticidas, metales pesados, tinturas y fármacos; y las hormonas, como los estrógenos.

Células y moléculas del sistema inmune. Están implicadas directamente en la pérdida de la tolerancia inmunológica, normalmente cumplen tareas de inmunovigilancia, que permiten discriminar entre lo propio y lo no propio, y activan la respuesta inmune contra lo que no se reconoce como propio, cuando se pierde la tolerancia:

De las células B, las B2 son la principal fuente de producción de auto anticuerpos y en la presentación de antígenos auto reactivos a las células $\mathrm{T}$, pueden ser inducidas por factores como los estrógenos y la prolactina. También podría existir la participación de las células B10 en la secreción de IL-10 y de las células B1 en menor grado, en la producción de autoanticuerpos reactivos (Selmi Leung et al., 2012).

De las células $\mathrm{T}$, las principalmente implicadas en los últimos años son las Th17 por la producción de IL-17 como mediador de inflamación crónica, inducidas por la señal de los Toll Like Receptors (TLR) de tipo 2. La asociación entre las artropatías inflamatorias y el gen de IL-17A/IL-17F especialmente con el polimorfismo rs 763780 ha sido recientemente confirmada, especialmente con AR y EA (Shao et al., 2020).

Las células Treg (células $T$ reguladoras) que normalmente son las responsables de la anergia periférica, serían responsables de la pérdida de la tolerancia inmu- nológica al estar disminuidas y presentar menor efectividad (Javinani et al., 2019; Selmi Leung et al., 2012).

En relación con las moléculas, las principales son los TLR, ya que reconocen patrones moleculares sobre la superficie de los patógenos, y en células auto reactivas pueden activar e inducir la producción de citocinas proinflamatorias como IL-6, TNF (factor de necrosis tumoral) e IFN (Javinani et al., 2019; Selmi Leung et al., 2012).

Cualquiera que sea el mecanismo, se requiere un individuo genéticamente susceptible y una importante desregulación del sistema inmune.

\section{Los genes en la patogenia de la ERA}

Se han reportado múltiples asociaciones genéticas, los principales de acuerdo con el papel que tendrían en la patogenia de estas enfermedades, según David y colaboradores (2018) y Javinani y colaboradores (2019) serían:

- Los genes del HLA-DR asociados directamente con la activación y función de linfocitos $\mathrm{T}$ y B auto reactivos.

- El gen Tumor Necrosis Factor Alpha Induced Protein 3 (TNFAIP3), está relacionado con la ruta de señalización e inducción de la síntesis del Tumor Necrosis Factor (TNF), a través de la activación del Nuclear Factor Kappa $\beta(\mathrm{NFk} \beta)$ y con participación directa en la respuesta inmune innata y en la respuesta inflamatoria.

- El gen Signal Transducer and Activator of Transcription 4 (STAT4), relacionado con la activación de células $\mathrm{T}$, la ruta de señalización e inducción de la síntesis de IFN y un aumento de la actividad de los TLR en la respuesta inmune innata y la activación de la respuesta celular.

- Los genes Interferón Receptor Factor (IRF) especialmente el tipo 5 (IRF5), están involucrados en la ruta de señalización e inducción de la síntesis del IFN y en el aumento de la actividad de los TLR, activando la respuesta inmune innata y la secreción de otras citocinas.

- El gen Integrin subunit Alpha M (ITGAM) relacionado con la fagocitosis y adhesión celular fundamentales en la eliminación de complejos inmunes.

- El gen Protein Tirosine Phosphatase non Receptor 22 (PTPN22), relacionado con la activación de linfocitos $\mathrm{T}$ auto reactivos. 
- El gen FC Gamma Receptor IIA (FCGRIIA) estaría relacionado con la deficiencia en la fagocitosis y la eliminación de complejos inmunes.

- Otros genes mencionados, pero aún menos comprendidos son el TNF-308A, PDCD1, BLKBANK1, TYK2 (Barturen \& Alarcón-Riquelme, 2018; Teruel \& Alarcón-Riquelme, 2016b).

- En los últimos años se ha confirmado la asociación de otros genes en el desarrollo de estas enfermedades:

- Los genes del complemento C4A y C4B han sido relacionados tanto con un efecto protector (C4A) y con un incremento del riesgo de desarrollar enfermedades como LES y Síndrome de Sjögren (C4B), el desequilibrio se ha asociado a la expresión de HLA DRB1*03:01 especialmente en hombres (Kamitaki et al., 2020).

- Los genes relacionados con el IFN y FOXP3 estarían involucrados con los cambios en la metilación del ADN, especialmente con la inactivación de genes que contribuirían a la desregulación del sistema inmune y la pérdida de la tolerancia en las principales ERA (De la Calle-Fabregat et al., 2020).

En la Tabla 1 se muestran ejemplos de estudios que han abordado la susceptibilidad de los principales genes encontrados y relacionados con ERA.

\section{Desarrollo de la susceptibilidad genética}

Se define a la susceptibilidad genética como el riesgo heredado de desarrollar una característica en particular, también puede entenderse como la carga genética que influye conjuntamente con el medio ambiente en el fenotipo de un organismo individual, y en el caso de las enfermedades, con un aumento en la probabilidad de desarrollar una en particular.

La presencia de un gen puede influir, pero no determina la presencia de una enfermedad. Puede ocurrir que exista un individuo con los genes de susceptibilidad, pero no desarrolle la enfermedad, se denomina penetrancia incompleta. Lo contrario, un individuo que desarrolla la enfermedad, pero no tiene los genes de susceptibilidad se le llama fenocopia (Laufer et al., 2017).

Las primeras descripciones sobre genes candidatos iniciaron hace tres décadas, y se ha documentado la asociación de genes tanto HLA: DR, DP y DQ, como no HLA: FCGRIIA, PTPN22, STAT, IRF5 y TYK2 utilizando técnicas de genotipificación individuales, que la mayoría de las veces utilizan como técnica a la Reacción de Cadena de Polimerasa (PCR por sus siglas en inglés), utilizando cebadores específicos; con el advenimiento de la tecnología para identificar eficazmente a los Polimorfismos de Nucleótido Simple (SNP) y la posibilidad de buscarlos en miles de muestras con técnicas modernas como la secuenciación de ADN o los Inmunochip, se abrió el camino a los estudios GWAS, que a partir del 2007 se han encargado a gran escala de confirmar los genes que ya existían, y de encontrar un número importante de nuevos genes, a los cuales aún no se les adjudica con propiedad ninguna implicación en la patogenia de estas enfermedades (Teruel \& Alarcón-Riquelme, 2016a)

El mejor diseño de investigación para cuantificar la susceptibilidad de un individuo a un gen relacionado con determinada enfermedad es el estudio de casos y controles; con el cálculo del riesgo "odds ratio" (OR) por sus siglas en inglés, además del establecimiento de las diferencias utilizando estadística paramétrica y no paramétrica.

La aparición de los GWAS y el análisis de miles de muestras genera la realización de revisiones especializadas: los metaanálisis (Pearson \& Manolio, 2008), que en los últimos años han sido la herramienta, para interpretar la expresión de múltiples genes y sus polimorfismos asociados a una enfermedad, y han brindado una mejor comprensión de las asociaciones existentes. A pesar de esto, los métodos utilizados en los GWAS requieren un estudio de validación, usualmente con métodos tradicionales (genotipificación individual por PCR), debido a los múltiples falsos positivos que pueden encontrarse (Eyre et al., 2017).

En la Figura 1 podemos ver en una línea de tiempo, los principales eventos que se han desarrollado en la búsqueda de genes en estas enfermedades.

Hoy en día puede medirse el riesgo que representa la presencia de un gen, a través del "Score Genético de Riesgo" (GRS por sus siglas en inglés), que se obtiene a partir de los GWAS y el cálculo de los OR y las frecuencias alélicas, de acuerdo con Belsky e Israel (2014) un riesgo alto incrementa la probabilidad del desarrollo de una enfermedad genéticamente determinada.

El cálculo del GRS recientemente se ha empezado a utilizar para predecir el daño orgánico (especialmente renal) y la mortalidad en estas enfermedades, y 
Tabla 1

Sinopsis de los principales estudios de la susceptibilidad genética en enfermedades reumáticas autoinmunes

\begin{tabular}{|c|c|c|c|c|c|}
\hline Gen & Enfermedad & Metodología & Población & Resultados & Referencias \\
\hline \multirow{11}{*}{$\begin{array}{l}\text { HLA } \\
\text { DRB1 }\end{array}$} & \multirow[t]{2}{*}{$\mathrm{AR}$} & \multirow{2}{*}{$\begin{array}{l}\text { Fine map } \\
\text { Estudio de asociación }\end{array}$} & Asiática & $\mathrm{p}=3.61 \times 10^{-112}$ & \multirow[t]{2}{*}{ Kim et al., 2016} \\
\hline & & & 2,234 & $>$ asociación & \\
\hline & \multirow[t]{2}{*}{ LES } & \multirow{2}{*}{$\begin{array}{l}\text { Fine map } \\
\text { Estudio de asociación }\end{array}$} & Asiática & $\mathrm{p}=5.64 \times 10^{-17}$ & \multirow[t]{2}{*}{ Kim et al., 2016} \\
\hline & & & 5,342 & > asociación & \\
\hline & \multirow[t]{2}{*}{ LES } & \multirow{2}{*}{$\begin{array}{l}\text { Uso de SNP } \\
\text { Estudio de casos/ } \\
\text { controles }\end{array}$} & Europea & OR 1.87 & \multirow[t]{2}{*}{ Morris et al., 2012} \\
\hline & & & 3,701 & $\mathrm{p}=.00001$ & \\
\hline & \multirow[t]{3}{*}{$\mathrm{AR}$} & \multirow{3}{*}{$\begin{array}{l}\text { metaanálisis } \\
\text { ( } 8 \text { estudios) } \\
\text { Estudio de asociación }\end{array}$} & Latinoamericana & OR 3.28 & \multirow{3}{*}{$\begin{array}{l}\text { Delgado-Vega et } \\
\text { al., } 2007\end{array}$} \\
\hline & & & 684 & $\mathrm{p}=.0001$ & \\
\hline & & & & & \\
\hline & \multirow[t]{2}{*}{ LES } & \multirow{2}{*}{$\begin{array}{l}\text { Estudio de casos y } \\
\text { controles }\end{array}$} & Latinoamericana & OR 2.14 & \multirow{2}{*}{$\begin{array}{l}\text { Castaño-Rodríguez } \\
\text { et al., } 2008\end{array}$} \\
\hline & & & 747 & $\mathrm{p}=.001$ & \\
\hline STAT 4 & \multirow[t]{4}{*}{ LES } & \multirow{4}{*}{$\begin{array}{l}\text { Uso de SNP } \\
\text { Estudio de casos y } \\
\text { controles }\end{array}$} & Latinoamericana & OR 2.34 & \multirow[t]{4}{*}{ Sánchez et al., 2010} \\
\hline ITGAM & & & 804 & $\mathrm{p}=.0001$ & \\
\hline IRF5 & & & & & \\
\hline PDCD1 & & & & & \\
\hline TNFAIP3 & \multirow[t]{3}{*}{ AR/LES } & Uso de SNP & & OR (TNFAIP3) 2.40 & \multirow[t]{3}{*}{ Ramos et al., 2011} \\
\hline IRF5 & & \multirow{2}{*}{$\begin{array}{l}\text { Estudio de casos y } \\
\text { controles }\end{array}$} & Europea & (IRF5) 1.97 & \\
\hline BLK & & & 1,500 & (BLK) 1.29 & \\
\hline \multirow[t]{2}{*}{ STAT 4} & \multirow[t]{2}{*}{$\mathrm{AR}$} & \multirow{2}{*}{$\begin{array}{l}\text { metaanálisis } \\
\text { ( } 21 \text { estudios) }\end{array}$} & Multiétnico & OR 1.56 & \multirow{2}{*}{$\begin{array}{l}\text { Ebrahimiyan et al., } \\
2019\end{array}$} \\
\hline & & & 15,732 & $\mathrm{p}=.0001$ & \\
\hline \multirow[t]{2}{*}{ PTPN22 } & \multirow[t]{2}{*}{ AR } & \multirow{2}{*}{$\begin{array}{l}\text { Estudio de asociación } \\
\text { de casos y controles }\end{array}$} & Caucásica & OR 1.65 & \multirow[t]{2}{*}{ Carlton et al., 2005} \\
\hline & & & 475 & $\mathrm{p}=.0006$ & \\
\hline HLA B27 & \multirow[t]{3}{*}{ EA } & \multirow{3}{*}{$\begin{array}{l}\text { Estudio de asociación } \\
\text { de casos y controles }\end{array}$} & Caucásica & OR (HLA B27) 0.75 & \multirow[t]{3}{*}{ Reveille et al., 2010} \\
\hline ERAP 1 & & & 2,053 & (ERAP 1) 1.36 & \\
\hline IL-23R & & & & (IL-23R) 1.32 & \\
\hline
\end{tabular}


Figura 1

Línea del tiempo de los hallazgos genéticos en ERA

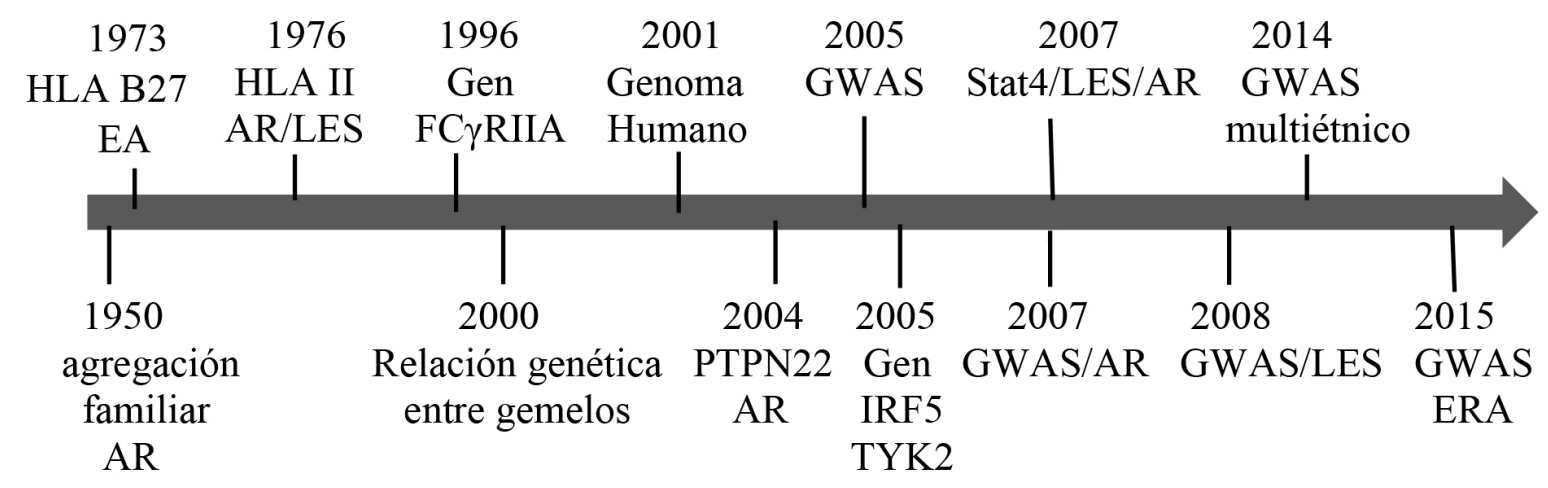

Nota . HLA $=$ Human Leucocyte Antigen, EA $=$ Espondilitis anquilosante, $\mathrm{AR}=$ Artritis reumatoide, LES $=$ lupus eritematoso sistémico, GWAS = Genome wide associatted study, ERA = enfermedad reumática autoinmune, PTPN22 $=$ protein tirosine phosphatase non receptor 22 , IRF5 $=$ Interferon receptor factor 5 , TYK2 $=$ Tirosin Kinase 2 , STAT4 $=$ signal transducer and activator of transcription 4 , Fc $\gamma$ RIIA $=$ Fcgamma receptor IIA.

en lupus eritematoso sistémico, se ha encontrado una relación directa entre un mayor GRS y un peor pronóstico (Reid et al., 2020).

Como se ha mencionado los avances tecnológicos permiten ahora una mayor discriminación de secuencias genéticas y las nuevas metodologías propuestas dirigirán la investigación genética, de acuerdo con varios grupos: Eyre y colaboradores (2017), Okada y colaboradores (2017) y Yukinori y colaboradores (2017) estas metodologías son:

Análisis EQTL. (expression quantitative trait loci): Son secuencias de ADN específicas, que permiten relacionar causalmente la expresión de uno varios genes.

Fine mapping. Facilita la identificación de todas las variantes de genes conocidas, en un locus especifico, lo que garantiza encontrar asociaciones fuertes. Es el complemento del GWAS.

Hi-C. Cuantifica interacciones entre varios fragmentos de ADN en un cromosoma y su par simultáneamente y no por separado, como habitualmente ocurre con PCR. Lo que mejora la causalidad.

Inmunochip. Es una herramienta para identificar SNP, ha permitido descubrir loci que representan riesgo para ERA.
Re-secuenciación. Complementa al fine mapping en la identificación de variantes no conocidas.

SNP genotyping array. Permite detector miles de SNP a través del genoma de manera simultánea.

\section{Susceptibilidad genética de las principales enfermedades reumáticas autoinmunes}

Susceptibilidad genética en AR. Esta enfermedad es considerada autoinmune debido a que en su patogenia se encuentran involucrados algunos auto anticuerpos, que se han relacionado con la presencia de algunos genes, desde el HLA DRB1 hasta más de 100 locus genéticos encontrados con las nuevas metodologías de análisis genético. Hoy se conoce gracias a los estudios GWAS que el $80 \%$ de la expresión genética ocurre en zonas no codificadas del ADN (Matzaraki et al., 2017; Okada et al., 2018).

Los genes podrían tener las siguientes implicaciones patogénicas: Activación de células T: PTNP22, CTLA4 y FOXP3; Diferenciación celular de T a Th1: STAT4; Facilitación de la citrulinización: PADI4 y HLA DRB1.

Se estima que en el $40 \%$ de los casos, se expresa el HLA DRB1, y con el advenimiento del concepto del epítopo compartido, se amplió el rango de variantes que explican el fenómeno. 
Se describe que los alelos DRB1*01, DRB $1 * 04$ y DBR $1 * 10$ presentan una importante asociación con la expresión de la enfermedad, DRB1*15 se asocia a una alta producción de anticuerpos para péptidos citrulinados (ACPA), mientras los alelos del epítopo compartido DEERA DRB1 *1301, *1302 y *1304 estarían asociados a un efecto protector para el desarrollo de AR (van der Helm-van Mil et al., 2007).

También se ha encontrado una estrecha relación entre la presencia de HLA DRB1, la sobrepoblación bacteriana y el desarrollo de AR ( $p=.00001)$ (Asquit et al., 2019), en apoyo a la hipótesis del mimetismo molecular asociado a la disbiosis del microbioma y la teoría del epítopo compartido, como activadores de células $\mathrm{T}$ e inductores del factor reumatoide y ACPA que pueden detectarse años antes de la expresión clínica de la enfermedad (Xu \& Yin, 2019).

El PTPN22 es otro de los genes que tiene una importante asociación con AR, el segundo después del HLA, y se ha relacionado por su papel en la codificación de la proteína linfoide tirosina fosfatasa, un regulador negativo de la activación de células $\mathrm{T}$, la presencia de un polimorfismo (rs 2476601) podría inhabilitar la codificación de esta proteína, aunque se desconoce la consecuencia de esto, se ha demostrado que su presencia incrementa el riesgo de desarrollo de la enfermedad (Carlton et al., 2005).

También se han encontrado polimorfismos de PTPN22 que han resultado ser protectores para el desarrollo de la enfermedad, como el caso de $-1123 \mathrm{G}>\mathrm{C}$, que se asocian con un nivel bajo de ACPA (Muñoz-Valle et al., 2017; Stahl et al., 2010).

Debe mencionarse el gen PADI4, involucrado por su participación en la codificación de la proteína peptidil arginina deaminasa 4, que convierte la arginina en residuos de citrulina (proceso de citrulinización), proceso ampliamente aceptado en la patogenia de la AR. Inicialmente descrito en población asiática, en el último tiempo se ha encontrado en la mayoría de las razas (Bax et al., 2011; Klareskog et al., 2006).

En los más de 100 loci que se han reportado, se mencionan asociaciones variables con otros genes: TRAF1, STAT4, FCGRIIA, CD40 y CCR6 (Karami et al, 2019; Okada et al., 2018).

El estudio de los micro ARN (miRNAs por sus siglas en inglés), ha sido proyectado como un biomarcador de utilidad en AR especialmente en etapas tempranas, consistentemente se ha relacionado a dos miRNAS (miR-155 y mir-146a) con el desarrollo de AR, Romo-García y colaboradores (Romo-García et al., 2019) en una población hispana en México, han reportado 97 miRNAs que podrían estar en relación con el desarrollo de artritis temprana, con especial significancia estadística el miR 361-5p que podría ser un marcador en el futuro.

También se ha investigado el tamaño de los telómeros, que han sido ligados al proceso inflamatorio y a la inmunidad adaptativa, y en el caso de AR algunos estudios han asociado los telómeros cortos con un incremento del riesgo; recientemente Zeng y colaboradores (2020) en un metaanálisis reporta que en pacientes con AR los telómeros son estadísticamente más cortos que los controles sanos, y los telómeros largos reducen el riesgo de desarrollar AR.

Susceptibilidad genética en LES. Es la enfermedad sistémica autoinmune por excelencia, caracterizada por la producción de auto anticuerpos que permiten el diagnóstico y seguimiento, y en cuya producción la presencia genética ha sido demostrada (Javinani et al., 2019). Los principales genes que han mostrado asociación son: HLA DRB1, FCGR, IRF5, STAT4 y en los últimos años PTPN22 y PDCD1 (Teruel \& Alarcón-Riquelme, 2016b).

Se han identificado aproximadamente 60 loci de riesgo para el desarrollo de LES, y a partir de 2007 que se desarrolló el primer GWAS, se confirmó la asociación de los genes ya mencionados, y se encontró asociación con otros genes: ITGAM, IRF7 y BLK-BLK1 (Crow, 2008; Harley et al., 2008; Hom et al., 2008). Sin embargo, la participación genética solo sería responsable del $30 \%$ de la heredabilidad de esta enfermedad (Hiraki \& Silverman, 2017).

Se ha propuesto que los genes pudieran estar implicados en la patogenia de la siguiente manera: Activación celular: células B, BLK y BANK1, célula T, PTPN22 y STATA 4, células dendríticas, PRDM1 y ETS1; Defectos en la apoptosis: TREX1, DNASE 1, DNASE1L3, FAS, FASL; Defectos del aclaramiento celular: deficiencias de C1q, C2 y C4; Inducción de la producción de citocinas y tráfico celular: IRF5, IRF7, IRF8, TNFAIP3 e ITGAM.

La asociación más importante reportada ha sido con el HLA DRB1*1501 y *0301 (Connolly \& Hakonarson, 2012; Godkhe-Puranik \& Niewold, 2015; Xue, 2018).

Los factores regulatorios del interferón (IRF), inducen la codificación de IFN que a su vez participan en la activación de los TLR en la respuesta inmune, de los nueve que se han descrito, tres tienen relevancia IRF5, IRF7 e IRF8. De estos el IRF5 es frecuentemente relacionado con los niveles elevados de IFN $\alpha$ que se 
encuentran en LES, los otros con menor asociación también se han reportado como mediadores en la activación de los TLR y la producción de IFN (Graham et al., 2007; Niewold et al., 2012).

El STAT 4 es otro gen que tiene una importante asociación, codifica a la proteína de transcripción 4 que participa en la actividad y producción de IFN $\alpha$, que esta presenta en la expresión de la enfermedad (Zheng et al., 2013).

El gen inductor de TNFAIP3 (proteína 3 inducible del tumor de necrosis alfa) participa en la codificación de la enzima A20, que regula NFKB, TNFa, TLR y el receptor de IL-1 (Wang et al., 2013).

El gen PTPN22 se ha relacionado con la presencia de dos polimorfismos, Arg620Trp (rs 2476601) que incrementa el riesgo de desarrollar LES y la variante Arg263Gln que tiene un efecto de protección para el desarrollo de la enfermedad (Stanford \& Bottini, 2014).

Otros genes con menor relevancia, pero que han sido identificados a partir de los GWAS son: BANK1, ITGAM cuya patogenia en LES se relaciona con la actividad de las células B y la expresión de moléculas de tráfico y adhesión celular (Bentham et al., 2015; Kozyrev et al., 2008; Nath et al., 2008).

Susceptibilidad en otras enfermedades reumáticas autoinmunes. Estas enfermedades comparten la expresión de genes, aunque no queda claro la participación que tienen en el desarrollo de éstas.

En la Espondilitis anquilosante (EA) se ha reportado una heredabilidad del $90 \%$ entre familiares de primer grado. Desde 1973 existe la asociación entre HLA B27 y el desarrollo de EA, sin embargo, una gran mayoría de individuos en quienes se ha encontrado el gen, no desarrollan la enfermedad (Hanson \& Brown, 2017; Osgood \& Knight, 2018).

En 2007 luego del GWAS se encontraron además del HLA B27, asociación con los genes ERA1, ERAP2 y genes que inducen el FNT y la IL-23. ERAP1 está involucrado en la expresión de la cadena de HLA B27 y en la síntesis de IL-17, mientras ERAP2 es un cofactor de expresión de ERAP1. La IL-23 a través de su receptor y señales JAK2 y STAT3 inducen la síntesis de IL-17 e IL-22, cuya participan en el proceso inflamatorio es relevante en esta enfermedad. Se induce la producción de TNF a través de sus genes TNFRFS1A, TRADD y TNFSF15, lo que facilita la perpetuidad del proceso inflamatorio (Burton et al., 2007).

El síndrome de Sjögren, regularmente se traslapa con genes de LES y AR, sin embargo, la mayoría de los reportes han encontrado asociación en: la producción de IFN a través de IRF5 y STAT 4, que también participa en la inducción de IL-12 y la diferenciación celular hacia Th1. La vía de activación de células $\mathrm{B}$, ha encontrado asociación con la expresión de BLK y la de células T con el gen CXCR5, el TNFa a través de los genes FNTAIP3 y TNIP1 (Teruel \& Alarcón-Riquelme, 2016b; Reksten et al., 2016).

Las miopatías inflamatorias (MI), son un grupo de heterogéneo de enfermedades autoinmunes que afectan a los músculos, se ha reportado asociación de estas con los genes HLA DRB1*03:01 y B*08:01, con Dermatomiositis DQB1 y PTNP22 con Polimiositis en europeos (Rothwell et al., 2016). En Japón se ha reportado asociación de MI con HLA DRB1*08:03 y DPB1*05:01 (Furukawa et al., 2020).

Susceptibilidad a las enfermedades reumáticas en América Latina. La expresión genética en América Latina es el producto de la mezcla de genes de origen europeo, amerindio y africanos, los datos reportados en varios países (Estados Unidos, Chile, Colombia, México, Perú, Argentina y Brasil), ha sido consistentes con lo reportado en otras latitudes, especialmente la asociación entre el HLA DRB1 y sus epítopos compartidos en AR y LES (Alarcón-Riquelme et al., 2016; Langefeld et al., 2017)

Se ha reportado asociaciones fuertes con los siguientes genes en AR, en poblaciones de Brasil, Colombia y México: STAT4, IRF5, IL2RA, PTPN22, y las noveles asociaciones entre: SPRED2, CCL21, ENOX1, NNA25 cuyo papel aun no es conocido (Castro-Santos \& Díaz-Peña, 2016; Delgado-Vega \& Anaya, 2007; López Herráez et al., 2013).

De manera aislada también se ha encontrado la expresión de PADI4 como factor de riesgo en el desarrollo de AR en mestizos de México (Baños \& Muñoz-Valle, 2017).

En LES también se ha encontrado una clara expresión de genes HLA DRB1, con la misma fuerza que se ha reportado en otras latitudes, mientras que genes no HLA reportados con una fuerte asociación fueron: STAT4, IRF5, ITGAM, PDCD1 y TNFSF4 (Alarcón-Riquelme et al., 2016; Castaño-Rodríguez et al., 2008; Sánchez et al., 2010).

Se ha generado discusión acerca del importante gen BLK, un activador de los linfocitos B y del gen BANK1 asociado también a la activación del linfocito $\mathrm{B}$, que se ha reportado en otras latitudes, pero en el estudio inicial en América Latina, no tuvo relevancia; reserva de lo reportado por Ramírez-Bello y colabo- 
radores, en una investigación recientemente realizada en población mestiza de México, han encontrado una asociación fuerte (Ramírez-Bello et al., 2019).

\section{Conclusiones}

La asociación genética en las ERA ha sido confirmada, y la susceptibilidad genética y las acciones biológicas inducidas por los genes involucrados, han sido ampliamente descritas; sin embargo, no existe un papel definido sobre su participación en la patogenia de estas enfermedades y se asumen varias posibilidades, entre las que destacan la inducción de citocinas proinflamatorias y la activación de células autorreactivas.

La expresión clínica de estas enfermedades es diversa, comparten entre ellas (con algunas variantes) genes y sus polimorfismos, y estas variaciones se han encontrado en la mayoría de los grupos raciales estudiados. Consistentemente se menciona a los genes del HLA DRB1, HLA B, PTPN22 y a los IRF (especialmente el IRF5) como los de mayor susceptibilidad genética.

La tecnología actual nos está acercando a definir con precisión a los genes que participan en el desarrollo de las ERA, y las propuestas para clasificarlas de acuerdo con la expresión genética son cada vez más aceptadas, lo que podría ser un impulso para la medicina de precisión.

\section{Conflicto de interés y Agradecimientos}

El autor declara no tener ningún conflicto de interés. Agradece la orientación en el proceso y la revisión del manuscrito al Lic. Armando Cáceres.

\section{Referencias}

Alarcón-Riquelme, M. E., Ziegler, J., Molineros, J., Howard, T. D., Moreno-Estrada, A., SánchezRodríguez, E., Ainsworth, H., Ortíz-Tello, P., Comeau, M., Rasmussen, A., Kelly, J., Adler, A., Acevedo-Vásquez, E., Cucho-Venegas, J., GarcíaDe la Torre, I., Cardiel, M., Miranda, P., Catoggio, L., Maradiaga-Ceceña, M., ... Jacob, C. (2016). GWAS in an Amerindian ancestry population reveals novel systemic lupus erythematosus risk loci and the role of European admixture. Arthritis \& Rheumatology, 68(4), 932-943. https://doi. org/10.1002/art.39504
Alarcón-Segovia, D., Alarcón-Riquelme, M. E., Cardiel, M. H., Caeiro, F., Massardo, L., Villa, A.R., Pons-Estel, B., \& Grupo Latinoamericano de Estudio del Lupus Eritematoso (GLADEL) (2005). Familial aggregation of systemic lupus erythematosus, rheumatoid arthritis, and other autoimmune diseases in 1,177 lupus patients from the GLADEL cohort. Arthritis \& Rheumatology, 52(4), 1138-1147. https://doi.org/10.1002/art.20999

Asquith, M., Sternes, P., Costello, ME., Karstens, L., Diamond, S., Martin, T., Li, Z., Marshall, M., Spector, T., Cao, K., Rosenbaum, J., \& Brown, M. (2019). HLA Alleles Associated with Risk of Ankylosing Spondylitis and Rheumatoid Arthritis Influence the Gut Microbiome. Arthritis \& Rheumatology, 71(10), 1642-1650. https://doi. org/10.1002/art.40917

Baños, C., \& Muñoz-Valle, J. (2017). PADI4 polymorphisms and the functional haplotype are associated with increased rheumatoid arthritis susceptibility: A replication study in a Southern Mexican population. Human Immunology, 78, 553-558. https://doi.org/10.1016/j humimm.2017.05.005

Barturen, G., \& Alarcón-Riquelme, M. E. (2018). Moving towards a molecular taxonomy of autoimmune rheumatic diseases. Nature Reviews Rheumatology, 14, 75-93. https://doi.org/10.1038/ nrrheum.2017.220

Bax, M., Van Heernst, J., Huizinga, T. W., \& Toes, R. E. (2011). Genetics of rheumatoid arthritis: what have we learned? Immunogenetics 63, 459-466. https://doi.org/10.1007/s00251-011-0528-6

Belsky D., \& Israel S. (2014). Integrating Genetics and Social Science: Genetic Risk Scores. Biodemography and Social Biology, 60, 137-155. https://doi.org/10.1080/19485565.2014.946591

Bentham J., Morris D. L., Graham, D. S. C., Pinder C. L., Tombleson P., Behrens T. W. Martin, J., Fairfax, B., Knight, J., Chen, L., Replogle, J., Syvänen, A., Rönnblom, L., Graham, R., Wither, J., Rioux, J., Alarcón-Riquelme, M. E., \& Vyse, T.J. (2015). Genetic association analyses implicate aberrant regulation of innate and adaptative immunity genes in the pathogenesis of systemic lupus erythematosus. Nature Genetic, 47, 1457-1464. https://doi. org/10.1038/ng.3434 
Burton, P., Clayton, D., Cardon, L., \& Wellcome Trust Case Control Consortium AustraloAnglo-American Spondylitis Consortium (TASC) (2007). Association scan of 14,500 nonsynonymus SNPs in four diseases identifies autoimmunity variants. Nature Genetics, 39(11), 1329-1337. https://doi.org/10.1038/ng.2007.17

Castaño-Rodríguez, N., Diaz-Gallo, L. M., PinedaTamayo, R., Rojas-Villarraga, A., \& Anaya, J. M. (2008). Meta-analysis of HLA-DRB1 and HLA-DQB1 polymorphisms in Latin American patients with systemic lupus erythematosus. Autoimmunity Reviews, 7, 322-330. https://doi. org/10.1016/j.autrev.2007.12.002

Castro-Santos, P., \& Díaz-Peña, R. (2016). Genetics of rheumatoid arthritis: a new boost is needed in Latin American populations. Revista Brasileira de Reumatología, 56(2), 171-177. https://doi. org/10.1016/j.rbre.2015.10.004

Carlton, V. E. H., Xu, H., Chokkalingam, A. P., Schrodi, S. J., Brandon, R., Alexander, H. C., Chang, M., Catanese, J., Leong, D., Ardlie, K., Kastner, D., Seldin, M., Criswell, L., Gregersen, P., Beasley, E., Thomson, G., Amos, C., \& Begovich, A.B. (2005). PTPN22 Genetic variation: Evidence for multiple variants associated with rheumatoid arthritis. American Journal of Human Genetics, 77, 567-581. https://doi.org/10.1086/468189

Connolly, J., \& Hakonarson, H. (2012). Role de cytokines in systemic lupus erythematosus: recent progress from GWAS and sequencing. Journal of Biomedicine and Biotechnology, 2012, 798924. https://doi.org/10.1155/2012/798924

Crow, M., (2008). Collaboration, genetic associations, and lupus erythematosus. New England Journal of Medicine, 358, 956-961. https://doi.org/10.1056/ NEJMe0800096

David, T., Ling, S., \& Barton, A. (2018). Genetics of immune-mediated inflammatory diseases. Clinical and Experimental Immunology, 193, 3-12. https://doi.org/10.1111/cei.13101

Deane, K., \& Carlson, E. (2012). Environmental and gene-environment interactions and risk of rheumatoid arthritis. Rheumatic Diseases Clinics of North American, 38(2), 405-426. https://doi. org/10.1016/j.rdc.2012.04.002
De la Calle-Fabregat, C., Morante-Palacios, O., \& Ballestar, E. (2020). Understanding the relevance of DNA methylation changes in immune differentiation and disease. Genes, 11(1), 110. https://doi.org/10.3390/genes 11010110

Delgado-Vega, A. M, \& Anaya, J. M. (2007). Metaanalysis of HLA-DRB1 polymorphism in Latin American patients with rheumatoid arthritis. Autoimmunity Reviews, 6, 402-408. https://doi. org/10.1016/j.autrev.2006.11.004

Ebrahimiyan, H., Mostafae, S., Aslani, S., Jamshidi, A., \& Mahmoudi, M. (2019). Studying the association between STAT4 gene polymorphism and susceptibility to rheumatoid arthritis disease: An updated meta-analysis. Iranian Journal of Immunology, 16(19),71-83. https://doi. org/10.22034/IJI.2019.39408

Eyre, S., Orozco, G., \& Worthington, J. (2017). The genetics revolution in rheumatology: large scale genomic arrays and genetic mapping. Nature Reviews Rheumatology, 13, 421-432. https://doi. org/10.1038/nrrheum.2017.80

Fernando, M. M. A., Stevens, C. R., Walsh, E. C., De Jager, P. L., Goyette, P., Plenge, R. M. Vyse, T., \& Riux, J. D. (2008). Defining the role of the MHC in autoimmunity: a reviewed and pooled analysis. PLoS Genetics, 4(4). https://doi.org/10.1371/ journal.pgen.1000024

Furukawa, H., Oka, S., Kawasaki, A., Hidaka, M., Shimada, K., Kondo, Y., Ihata, A., Matsushita, T., Matsumoto, T., Komiya, A., Kobayashi, K., Osada, A., Katayama, M., Okamoto, A., Setoguchi, K., Kono, H., Hamaguchi, Y., Matsui, T., Fukui, N., ... Thoma, S. (2020). Human leukocyte antigen in Japanese patients with idiopathic inflammatory myopathy. Modern Rheumatology, 30(4), 696-702. https://doi.org/10 $.1080 / 14397595.2019 .1637593$

Godkhe-Puranik, Y., \& Niewold, T. (2015). Immunogenetics of systemic lupus erythematosus: A comprehensive review. Journal of Autoimmunity, 64, 125-136. https://doi.org/10.1016/j. jaut.2015.08.004

Graham, R. R., Kyogoku, C., Sigurdsson, S., Vlasova, I. A., Davies, L. R. L., Baechler, E. C., Plenge, R., Koeuth, T., Ortmann, W., Hom, G., Bauer, J., 
Gillett, C., Burtt, N., Cunninghame, D., Onofrio, R., Petri, M., Gunnarsson, I., Svenungsson, E., Rönnblom, L., ... Altshuler D. (2007). Three functional variants of IFN regulatory factor 5 (IRF5) define risk and protective haplotypes for human lupus. Proceedings of the National Academy of Sciences of the United States of America, 104, 6758-6763. https://doi.org/10.1073/ pnas.0701266104

Hanson, A., \& Brown, M. (2017). Genetics and the causes of ankylosing spondylitis. Rheumatic Diseases Clinics of North American, 43, 401-414. https://doi.org/10.1016/j.rdc.2017.04.006

Harley, J., Alarcón-Riquelme, M. E., Criswell, L., Jacob, C., Kimberly R., \& Moser, K. (2008). Genome wide association scan in women with systemic lupus erythematosus identifies susceptibility variants in ITGAM, PXK, KIAA1542 and other loci. Nature Genetics, 40, 204-210. https://doi. org/10.1038/ng.81

Hiraki, L., \& Silverman, E. (2017). Genomics of systemic lupus erythematosus: Insights Gained by studying monogenic young onset. Rheumatic Diseases Clinics of North American, 43, 415-434. https://doi.org/10.1016/j.rdc.2017.04.005

Hom, G., Graham, R. R., Modrek, B., Taylor, K. E., Ortmann, W., Garnier, S., Lee, A., Chung, S., Ferreira, R., Pant, K., Ballinger, D., Kosoy, R., Demirci, Y., Ilyas, M., Kao, A., Tian, C., Gunnarsson, I., Bengtsson, A., RantapääDahlqvist, S., Petri, M., ... Behrens T.W. (2008). Association of systemic lupus erythematosus with C8orf13-BLK and ITGAMITGAX. New England Journal of Medicine, 358, 900-909. https://doi.org/10.1056/NEJMoa0707865

Javinani, A., Ashraf-Ganjouei, A., Aslani, S., Jamshidi, A., \& Mahmoudi, M. (2019). Exploring the etiopathogenesis of systemic lupus erythematosus: a genetic perspective. Immunogenetics, 71(4), 283-297. https://doi. org/10.1007/s00251-019-01103-2

Kamitaki, N., Sekar, A., Handsaker, R., de Rivera, H., Tooley, K., Morris, D.L., Taylor, K., Whelan, C., Tombleson, P., Olde, L., Boehnke, M., Kimberly, R., Kaufman, K., Harley, J., Langefeld, C., Seidman, C., Pato, M., Pato, C., Ophoff, R., Graham, R., ... McCarroll, S. A.
(2020). Complement genes contribute sex-biased vulnerability in diverse disorders. Nature, 582, 577.581. https://doi.org/10.1038/s41586-0202277-x

Karami, J., Aslani, S., Jamshidi, A., Garhasbi M., \& Mahmoudi, M. (2019). Genetic implication in the pathogenesis of rheumatoid arthritis; an updated review. Gene, 702, 8-16. https://doi.org/10.1016/j. gene.2019.03.033

Kim, K., Bang, S.-Y., Hyun Yoo, D., Cho, S.-K., Choi, C.-B., Sung, Y.-K., Kim, T.-H., Jun, J.-B., Kang, Y., Suh, C.-H., Shim, S.-C., Lee, S.-S., Lee, J., Chung, W., Kim, S.-K., Choe, J.-Y., Nath, S., Lee, H.-S., \& Bae, S.-C. (2016). Imputing variants in HLA-DR beta genes reveals that HLA-DRB1 is solely associated with rheumatoid arthritis and systemic lupus erythematosus. PLOS ONE, 11(2), e0150283. https://doi.org/10.1371/journal. pone. 0150283

Klareskog, L., Padyukov, L., Lorentzen, J., \& Alfredsson, L. (2006). Mechanisms of disease: Genetic susceptibility and environmental triggers in the development of rheumatoid arthritis. Nature Review Rheumatology, 2, 425-433. httpps://doi.org/10.1038/ncprheum0249

Kozyrev, S., Abelson, A., Wojcik, J., Zaghlool, A., Linga, M., Sánchez, E., Gunnarsson, I., Svenungsson, E., Sturfelt, G., Jönsen, A., Truedsson, L., Pons-Estel, B., Witte, T., D'Alfonso, S., Barizzone, N., Danieli, M.-G., Gutierrez, C., Suarez, A., Junker, P., ... AlarcónRiquelme, M. E. (2008). Functional variants in the B cell gene BANK1 are associated with systemic lupus erythematosus. Nature Genetics, 40, 211-216. https://doi.org/10.1038/ng.79

Kuo, C.-F., Grainge, M., Valdes, A., See, L.-C., Yu, K.H., Shaw, S., Lou, S.-F., Zhang, W., \& Doherty, M. (2017). Familial aggregation of rheumatoid arthritis and co-aggregation of autoimmune diseases in affected families: A nationwide population-based study. Rheumatology, 56, 928-933. https://doi.org/10.1093/rheumatology/ kew500

Langefeld, C.D., Ainsworth, H., Cunninghame, D., Kelly, J., Comeau, M., Marion, M., Howard, T., Ramos, P., Croker, J., Morris, D., Sandling, J., Carlsson, J., Acevedo-Vásquez, E., Alarcón, 
G., Babini, A., Baca, V., Bengtsson, A., De la Rúa Figueroa, I., Doria, A., ... Vyse, T. (2017). Transancestral mapping and genetic load in systemic lupus erythematosus. Natural Communications, 8,16021 . https://doi. org/10.1038/ncomms16021

Laufer, V., Chen, J., Langefeld, C., \& Bridges, L. (2017). Integrative approaches to understanding the pathogenic role of genetic variation in rheumatic diseases. Rheumatic Diseases Clinics of North American, 43(3), 449-466. https://doi. org/10.1016/j.rdc.2017.04.012

López Herráez, D., Martínez-Bueno, M., García de la Torre, I., Sacnún, M., \& Goñi, M. (2013). Rheumatoid arthritis in Latin Americans enriched for Amerindian ancestry is associated with loci in chromosomes 1, 12 and 13, and the HLA class II region. Arthritis \& Rheumatology, 65(6), 1457-1467. https://doi.org/10.1002/art.37923

Matzaraki, V., Kumar, V., Wijmenga, C., \& Zhernakova, A. (2017). The MHC locus and genetic susceptibility to autoimmune and infectious diseases. Genome Biology, 18, 76, 2-21. https://doi.org/10.1186/s13059-017-1207-1

Morris. D., Taylor, K., Fernando, M., Nititham, J., Alarcón-Riquelme, M. E., Barcellos, L., Behrens, T., Cotsapas, C., Gaffney, P., Graham, R., PonsEstel, B., Gregersen, P., Harley, J., Hauser, S., Hom, G., Langefeld, G., Noble, J., International MHC and Autoimmunity Genetics Network \& Vyse T. (2012). Unraveling multiple MHC gene associations with systemic lupus erythematosus: Model choice indicates a role for HLA alleles and non-HLA genes in Europeans. American Journal of Human Genetics 91, 778-793. https://doi.org/10.1016/j.ajhg.2012.08.026.

Moser, K., Kelly, J., Lessard, C., \& Harley, J. (2009). Recent insights into the genetic basis of systemic lupus erythematosus. Genes Immunology, 10, 373-379. https://doi.org/10.1038/gene.2009.39

Muñoz-Valle, J., Padilla-Gutiérrez, J., HernándezBello, J., Ruiz-Noa, Y., Valle, Y., PalafoxSánchez, C., Parra-Rojas, I., Gutiérrez-Ureña, S., \& Rangel-Villalobos, H. (2017). Polimorfismo $-1123 \mathrm{G}>\mathrm{C}$ en el gen PTPN22 y anticuerpos antipéptido citrulinado cíclico en la artritis reumatoide. Reumatología Clínica, 149(3), 95100. https://doi.org/10.1016/j.medcli.2017.01.025
Nath, S., Han, S., Kim-Howard, X., Kelly, J., Viswanathan, P., Gilkeson, G., Chen, W., Zhu, C., McEver, R., Kimberly, R., Alarcón-Riquelme, M.-E., Vyse, T., Li, Q.-Z., Wakeland, E., Merrill, J., James, J., Kaufman, K., Guthridge, J., \& Harley, J. (2008). A nonsynonymous functional variant in integrin-alpha (M) encoded by ITGAM is associated with systemic lupus erythematosus. Nature Genetics, 40, 152-154. https://doi. org/10.1038/ng.71

Niewold, T., Kelly, J., Kariuki, S., Franek, B., Kumar, A., Kaufman, K., Thomas, K., Walker, D., Kamp, S., Frost, J., Wong, G., Merrill, J., AlarcónRiquelme, M.-E., Tikly, M., Ramsey-Goldman, R., Reveille, J., Petri, M., Edberg, J., Kimberly, P., ... Harley, J. B. (2012). IRF5 haplotypes demonstrate diverse serological associations which predict serum interferon alpha activity and explain the majority of the genetic association with systemic lupus erythematosus. Annals of the Rheumatic Diseases, 71(3), 463-468. https://doi. org/10.1136/annrheumdis-2011-200463

Okada, Y., Kishikawa, T., Sakaue, S., \& Hirata, J. (2017). Future directions of genomics in rheumatic diseases. Rheumatic Diseases Clinics of North American, 43(3), 481-487. https://doi. org/10.1016/j.rdc.2017.04.009

Okada, Y., Eyre, S., Suzuki, A., Kochi Y., \& Yamamoto, K. (2018). Genetic of rheumatoid arthritis: 2018 status. Annals of Rheumatic Diseases, 78, 446-473. https://doi.org/10.1136/ annrheumdis-2018-213678.

Osgood, J. E., \& Knight, J. C. (2018). Translating GWAS in rheumatic disease: Approaches to establishing mechanism and function for genetic associations with ankylosing spondylitis. Briefing in Functional Genomics, 17(5), 308-318. https:// doi.org/10.1093/bfgp/ely015

Pearson, T. A., \& Manolio, T. A. (2008). How to interpret a genome wide association study. Journal American Medical Association, 299(11), 1335-1344. https://doi.org/10.1001/ jama.299.11.1335

Ramírez-Bello, J., Jiménez-Morales, S., MontufarRobles, I., Fragoso, J., Barbosa-Cobos, R. E., Saavedra, M. A., \& Sánchez-Muñoz, F. (2019). BLK and BANK1 polymorphisms and interactions are associated in Mexican patients 
with lupus erythematosus. Inflammation Research, 68, 705-713. https://doi.org/10.1007/ s00011-019-01253-9

Ramos, P., Criswell, L. A., Moser, K. L., Comeau, M. E., Williams, A. H., Pajewski, N. M., Chung, S., Graham, R., Zidovetzki, R., Kelly, J., Kaufman, K., Jacob, C., Vyse, T., Tsao, B., Kimberly, R., Gaffney, P., Alarcón-Riquelme, M.-E., Harley, J., Langefeld, C., \& International Consortium of the Genetics of Systemic Erythematosus Lupus (SLEGEN). (2011). A comprehensive analysis of shared loci between systemic lupus erythematosus and sixteen autoimmune diseases reveals limited genetic overlap. PLoS Genetics, 7(12), e1002406. https://doi.org/10.1371/ journal.pgen.1002406

Reid, S., Alexsson, A., Frodlund, M., Morris, D., Sandling, J. K., Bolin, K., Svenungsson, E., Jönsen, A., Bengtsson, C., Gunnarsson, I., Illescas Rodriguez, V., Bengtsson, A., Arve, S., Rantapää-Dahlqvist, S., Eloranta, M.-L., Syvänen, A.-C., Sjöwall, C., Vyse, T., Rönnblom, R., \& Leonard, D. (2020). High genetic risk score is associated with early disease onset, damage accrual and decreased survival in systemic lupus erythematosus. Annals of Rheumatic Diseases, 79, 363-369. https://doi.org/10.1136/ annrheumdis-2019-216227

Reksten, T. R., Lessard, C. J., \& Sivils, K. L. (2016). Genetics in Sjögren syndrome. Rheumatic Diseases Clinics of North American, 42, 435-447. https://doi.org/10.1016/j.rdc.2016.03.003

Reveille, J., Sims, A., Danoy, P., Evans, D., Leo, P., Pointon, L., Jin, R., Zhou, X., Bradbury, L., Appleton, L., Davis, J., Diekman, L., Doan, T., Dowling, A., Duan, R., Duncan, E., Farrar, C., Hadler, J., Harvey, D., ... Brown, M. [The Australo-Anglo-American Spondyloarthritis Consortium (TASC)]. (2010). Genome-wide association study of ankylosing spondylitis identifies non-MHC susceptibility loci. Nature Genetics, 42(2), 123-127. https://doi.org/10.1038/ ng.513
Romo-García, M.F., Bastian, Y., Zapata-Zúñiga, M., Macías-Segura, N., Castillo-Ortiz, J.D., LaraRamírez, E.E., Fernández-Ruiz, J., Berlanga. Taylor, A., González-Amaro, R., Ramos-Remus, C., Enciso-Moreno, J., \& Castañeda-Delgado, J.E. (2019). Identification of putative miRNA biomarkers in early rheumatoid arthritis by genome-wide microarray profiling: A pilot study. Gene, 720, 144081. https://doi.org/10.1016/j. gene.2019.144081

Rothwell, S., Cooper, R.G., Lundberg, I. E., Miller, F.W., Gregersen, P. K., Bowes, J., Vencosvsky, J., Danko, C., Lymaye, V., Selva-O'Callaghan, A., Hanna, M., Machado, P., Pachman, L., Reed, A., Rider, L., Cobb, J., Platt, H., Molberg, O., Benveniste, O., Mathiessen, P., ... Lamb, J. (Myositis Genetic Consortium). (2016). Dense genotyping of immune-related loci in idiopathic inflammatory myopathies confirms HLA alleles as the strongest genetic risk factor and suggests different genetic background for major clinical subgroups. Annals of the Rheumatic Diseases, 75(8), 1558-66. https://doi.org/10.1136/ annrheumdis-2015-208119

Sánchez, E., Webb, R. D., Rasmussen, A., Kelly, J. A., Riba, L., Kaufman, K. M., García-de la Torre, I., Moctezuma, J., Maradiaga-Ceceña, M., Cardiel-Rios, M., Acevedo, E., CuchoVenegas, M., García, M., Gamron, S., Pons-Estel, B., Vasconcelos, C., Martin, J., Tusié-Luna, T., Harley, J., ... Alarcón-Riquelme, M. E. (2010). Genetically determined Amerindian ancestry correlates with increased frequency of risk alleles for systemics lupus erythematosus. Arthritis \& Rheumatology, 62(12), 3722-3729. https://doi. org/10.1002/art.27753

Selmi, C., Leung, P. S. C., Sherr, D. S., Diaz, M., Nyland, J. F., Monestier, M., Rose, N., \& Gershwin, M. E. (2012). Mechanism of environmental influence on human autoimmunity: A national institute of environmental health sciences expert panel workshop. Journal of Autoimmunity, 39, 282-284. https://doi.org/10.1016/j.jaut.2012.05.007 
Selmi, C., Lu, Q., \& Humble, M. C. (2012). Heritability versus the role of the environment autoimmunity. Journal of Autoimmunity, 39, 249-252. https://doi. org/10.1016/j.jaut.2012.07.011

Shao, M., Xu, S., Yang, H., Xu, W., Deng, J., Chen, Y., Gao, X., Guan, S., Xu, S., Shuai, Z., \& Pan, F. (2020). Association between IL-17A and IL$17 \mathrm{~F}$ gene polymorphism and susceptibility in inflammatory arthritis: A meta-analysis. Clinical Immunology 213, 108374. https://doi.org/10.1016/j. clim.2020.108374

Sisó-Almirall, A., Kostov, B., Martínez-Carbonell, E., Brito-Zerón, P., Blanch Ramirez, P., AcarDenizli, N., Delicado, P., González-Martínez, S., Velasco Muñoz, C., Benavent Áreu, J., \& Ramos-Casals, M. (2020). The prevalence of 78 autoimmune diseases in Catalonia (MASCATPADRIS Big Data Project). Autoimmunity Reviews, 19(2), 102448. https://doi.org/10.1016/j. autrev.2019.102448

Stahl, E. A, Raychaudhuri, S., Remmers, E. F., Xie, G., Eyre, S., Thomson B. P., Li, Y., Kurreeman, F., Zhernakova, A., Hinks, A., Guiducci, C., Chen, R., Alfredsson, L., Amos, C., Ardlie, K., Barton, A., BIRAC Consortium., Bowes, J., Burtt, N., ... Plenge, R. M. (2010). Genome wide association study meta-analysis identified seven new rheumatoid arthritis risk loci. Nature Genetics, 42, 508-514. https://doi.org/10.1038/ng.582

Stanford, S. M., \& Bottini, N. (2014). PTPN22: the archetypal non-HLA autoimmunity gene. Nature Review Rheumatology, 10, 602-611. https://doi. org/10.1038/nrrheum.2014.109

Teruel, M., \& Alarcón-Riquelme, M. E. (2016a). The genetic basis of systemic lupus erythematosus: What are the risk factors and what have we learned. Journal of Autoimmunity, 74, 161-175. https://doi.org/10.1016/j.jaut.2016.08.001

Teruel, M., \& Alarcón-Riquelme, M. E. (2016b). Genetics of systemic lupus erythematosus and Sjögren's syndrome: an update. Current Opinion Rheumatology, 28(5), 506-514. https://doi. org/10.1097/BOR.0000000000000310 van der Helm-van Mil, A., Verpoort, K., Le Cessie, S., Huizinga, T., De Vries, R., \& Toes, R. (2007). The HLA-DRB1 shared epitope alleles differ in the interaction with smoking and predisposition to antibodies to cyclic citrullinated peptide. Arthritis \& Rheumatology, 56, 425-432. https://doi.org/10.1002/art. 22373

van Drongelen, V., \& Holoshitz. J. (2017). Human leukocyte antigen disease associations in rheumatoid arthritis. Rheumatic Diseases Clinics of North American, 43(3), 363-376. https://doi. org/10.1016/j.rdc.2017.04.003

Wang, S., Wen, F., Wiley, G., Kinter, M., \& Gaffney, P. (2013). An enhancer element harboring variant associated with systemic lupus erythematosus engages the TNFAIP 3 promote to influence A20 expression. PLoS Genetics, 9, Article e1003750. https://doi.org/10.1371/journal.pgen.1003750

$\mathrm{Xu}$, H., \& Yin, J. (2019). HLA risk alleles and gut microbiome in ankylosing spondylitis and rheumatoid arthritis. Best Practice \& Research Clinical Rheumatology, 33(6),101499. https://doi. org/10.1016/j.berh.2020.101499

Xue, K., Niu, W., \& Cui, Y. (2018). Association of HLA-DR3 and HLA-DR15 Polymorphisms with Risk of Systemic Lupus Erythematosus. Chinese Medical Journal, 131(23), 2844-51. https://doi. org/10.4103/0366-6999.246058

Yukinori, O., Kishikawa, T., Sakaue, S., \& Hirata, J. (2017). Future directions of genomics research in rheumatic diseases. Rheumatic Diseases Clinics of North American, 43(3), 481-487. https://doi. org/10.1016/j.rdc.2017.04.009

Zeng, G., Zang, W., Qian, Y., Huang, H., Wu, D., He, Z., Ye, D., Mao, Y., \& Wen, C. (2020). Association of telomere length with risk of rheumatoid arthritis: a meta-analysis and Mendelian randomization. Rheumatology, 59(5), 940-947. https://doi. org/10.1093/rheumatology/kez524

Zheng, J., Yin, J., Huang, R., Peterson, F., \& Yu, X. (2013). Meta-analysis reveals an association of STAT4 polymorphisms with systemic autoimmune disorders and anti dsDNA antibody. Human Immunology, 74, 986-992. https://doi. org/10.1016/j.humimm.2013.04.034 